\title{
Integration of shipment scheduling decisions for forward and reverse channels in a recoverable item system
}

\author{
Ayșegül Toptal \\ Industrial Engineering Department, Bilkent University, Ankara 06800, Turkey
}

\section{A R T I C L E I N F O}

Article history:

Received 29 October 2010

Accepted 21 June 2011

Available online 3 July 2011

Keywords:

Recoverable item

Spare inventory

Transportation

Cargo capacity

\begin{abstract}
A B S T R A C T
In this paper, we consider the problem of finding the economic shipment quantities of failed and recovered items between a central depot and a collection center to coordinate the flow in both ways. Our model takes an explicit account for the transportation costs and capacities under the assumption of deterministic failure rate of items. The proposed solution provides the optimum shipment quantities, and the level of spare items to be held at the collection center with the objective of minimizing the long-run average total costs subject to a service level.
\end{abstract}

(c) 2011 Elsevier B.V. All rights reserved.

\section{Introduction and literature}

In recent years, reuse of recoverable items has gained importance due to increased awareness of environmental issues and economic benefits. Collection of used/returned items, their testing and sorting, recovery operations (e.g., repair, remanufacturing, recycling) and redistribution are some of the activities that take place in recoverable product environments. In this paper, we study the integration of shipment decisions concerning the forward and reverse channels between a collection center/local warehouse and a central depot/recovery location.

In the system that is being studied, items that are used at several bases/retail locations are returned to a collection center upon failure. Failed items are consolidated at the collection center until an economic quantity is reached and shipped to a central depot for recovery. The collection center is also used for storing spare items that can be put into service to replace some of the failed ones. Testing, sorting and recovery operations of the failed items are performed at the central depot. If a failed item cannot be recovered, it is replaced with a new product. The inventory of spare items at the collection center and the awaiting users of failed items are replenished from the central depot periodically. The shipment of items between the collection center and the central depot, in both ways, is done using capacitated vehicles. In this setting, we study the problem of finding the optimum amount of spare items at the collection center, economic shipment quantity of failed items from the collection center to the central depot and economic shipment quantity of serviceable

E-mail address: toptal@bilkent.edu.tr items (i.e., recovered or replaced) from the central depot to the collection center. Our objective is to minimize costs while achieving a service level over all bases/retail locations.

The problem of interest is motivated by the practice of a beverage company in Turkey. This company provides refrigerators to the retailers that sell the company's products. The company holds the ownership of these refrigerators and handles their recovery upon failure. The retailers that are in close proximity are grouped. Each group is assigned a warehouse for holding spare refrigerators and storing those that need to be recovered after failure. As the number of failed refrigerators accumulates to a certain level, a shipment is scheduled from the central location to the warehouse for picking them up. The same shipment is also utilized for carrying spare refrigerators from the central location to the warehouse. At the central location, refrigerators collected from various groups of retailers are examined to identify their cause of failure. As a result, a refrigerator is either repaired, remanufactured or disposed. Repair brings a refrigerator to a working condition through cleaning and correcting the faults. Remanufacturing involves a greater degree of work such as changing the evaporator, painting, etc., and brings the refrigerator to an equivalent quality level to that of a new one. A disposed refrigerator is replaced with a brand new one. The cycle of inventory replenishment of recovered (repaired, remanufactured or replaced) refrigerators at the warehouse and pick up of those that have failed, continues with the next shipment. A retailer, whose refrigerator fails, is provided a new refrigerator if the spares inventory has one, otherwise, he/she waits until the next scheduled shipment to the area.

In the current study, we focus on the problem of determining the frequency of shipments to and from a local warehouse and the 
level of spare items to be held at this location. We estimate the accumulation rate of failed refrigerators at the warehouse using a deterministic and constant value $(\lambda)$. This may provide a good approximation to the actual failure rate if the percentages of repaired, remanufactured or replaced refrigerators being used by retailers at the current group are almost the same at all times. This study does not take into account the inspection/separation procedures or the purchasing decisions at the central warehouse. Instead, the main focus is on the collection and redistribution. The motivation for ordering in batches and holding some spare items at the local warehouse stems from reducing transportation costs and waiting costs of users.

For transporting failed items from the local warehouse to the central depot and recovered items in the opposite direction, capacitated vehicles/trucks are used. A truck has a capacity of carrying $P$ items, and costs a value of $R$ regardless of its percentage capacity utilized. This form of freight cost is known as the multiple-setups cost structure, and is commonly used in the literature on integrated inventory and transportation decisions for modeling full truckload shipments. The focus of the studies in this area is to find the optimal replenishment quantities that minimize the joint inventory and transportation costs. Aucamp (1982), Lee (1989), and Toptal (2009) are some examples of papers that consider full truckload (FTL) shipments in single-echelon inventory replenishment problems. Several recent papers propose integrated models for joint replenishment and transportation decisions in multi-echelon inventory systems (see Cetinkaya and Lee, 2002; Toptal et al., 2003; Toptal and Çetinkaya, 2006). We note that there are also studies that take into account less than truckload (LTL) shipments (Burwell et al., 1997; Tersine and Barman, 1994) or a combination of TL and LTL transportation (Mendoza and Ventura, 2008; Rieksts and Ventura, 2010).

Another body of research that is related to the current paper concerns recoverable item inventory systems. A group of studies in this area focus on the operating policies of a supplier who satisfies demand either by newly manufactured products or repaired old products. These studies typically assume deterministic demand and return rates, and investigate the optimal repair lot sizes and production lot sizes to minimize costs (Schrady, 1967; Nahmias and Rivera, 1979; Richter, 1996a, 1996b; Teunter, 2001; Koh et al., 2002). Also related to our study is the stream of research on repair and spare parts models, pioneered by Sherbrooke (1968). A common objective of these models is to find the spare inventory levels at the depot and/or the bases in a system where items being used at the bases can be repaired either at this level or at the central depot (see Sherbrooke, 1968; Moinzadeh and Lee, 1986; Kim et al., 2000). Spare inventory is held at several locations to minimize expected shortage cost and to satisfy some service level during the repair time of the failed items. For extensive reviews of these models, we refer to Guide and Srivastava (1997) and Kennedy et al. (2002).

We note that, although there is a rich literature in the general area of recoverable item inventory systems, to our knowledge, there is no study that models transportation costs and capacities. However, significant savings can be realized if transportation costs are also considered along with shortage and inventory holding costs. This study explicitly accounts for the usage of a capacitated vehicle and coordinates the shipment scheduling decisions between a central depot and a collection center through finding the economic shipment quantities of recovered and failed items to be carried with the same dispatch of a vehicle. In the next section, we begin with defining and formulating the problem. Section 3 presents the analysis of the problem and its solution. Some experimental results and implementation of the solution are discussed in Section 4.

\section{Problem definition and notation}

We consider a company that manages the operations related to the recovery of failed items at several locations. Failed items at these locations, which are in close proximity, are stored temporarily at a local warehouse until they are shipped to a central location for their recovery. The local warehouse has ample capacity and is also used for holding spare items (see Fig. 1). The replenishment of spare items is done periodically from the central location using a capacitated vehicle. On the way back from the local warehouse to the central location, the same vehicle is also utilized for carrying the failed items. At the time of a failure at a user/retailer, if there is a spare item at the warehouse, it is used to replace the failed item. If the warehouse is out of stock for spare items, the user of the failed item has to wait until the next replenishment of the local warehouse. In the latter case, the company incurs a cost for each time unit that the retailer waits. At the central location, failed items are either recovered or disposed. If a failed item is disposed, it is replaced by a brand new one.

The locations that are served through a warehouse are in close proximity. The company may have several other groups of locations with their own warehouses. However, the focus of this paper is to study the shipment scheduling and replenishment of the warehouse associated with a single group of locations. We consider a case in which the company wants the level of failed items which cannot be immediately replaced in each cycle, not to exceed a predetermined value $k$. This is primarily for achieving a

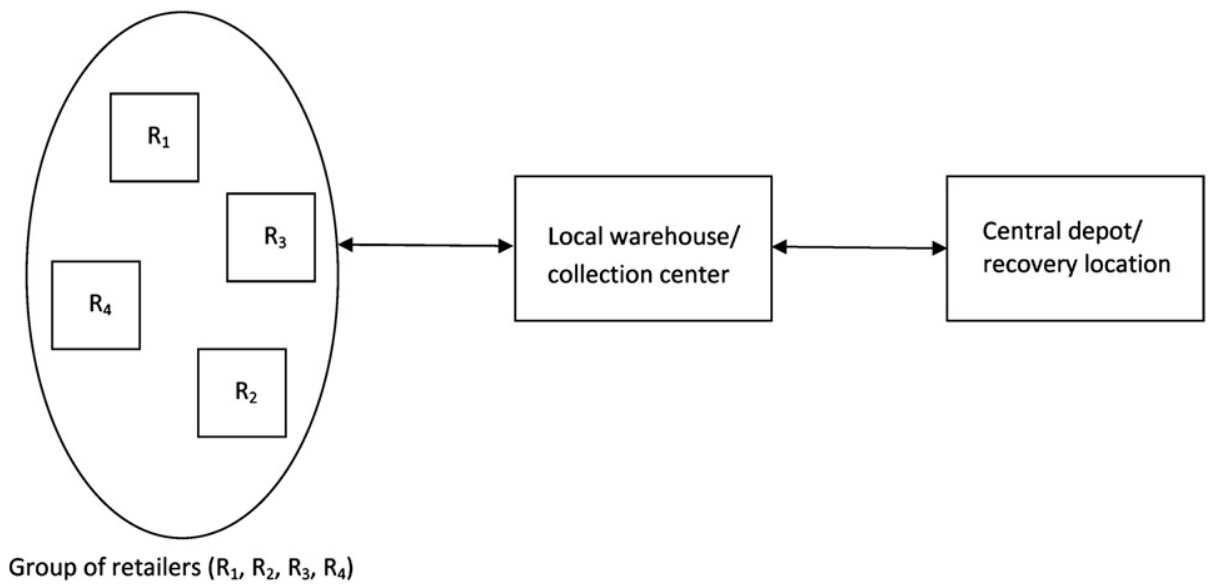

Fig. 1. An illustration of the inventory locations at the local region and the central location. 
Table 1

Notation.

\begin{tabular}{ll}
\hline$T$ & Length of the shipment cycle \\
$n$ & Quantity of items in use at all locations of the current group \\
$h_{1}$ & Cost of holding one unit of spare item at the warehouse for a unit time \\
$h_{2}$ & Cost of holding one unit of a failed item at the warehouse for a unit time \\
$w$ & Cost per unit time incurred by a user for waiting without an operating item \\
$\lambda$ & Failure rate of items over all the locations at the current group \\
$m$ & Level of spare items at the warehouse at the beginning of each cycle $(m \geq 0)$ \\
$k$ & Maximum level of failed items in each cycle which cannot be immediately replaced $(k \ll n$ and $k \leq P)$ \\
$Q$ & Quantity of items shipped from the central location to the warehouse at each cycle $(Q>0)$ \\
$P$ & Capacity of a truck \\
$R$ & Cost per truck \\
$T C(Q, m)$ & Long-run average total cost as a function of $Q$ and $m$ \\
\hline
\end{tabular}

certain level of customer satisfaction. We assume that the value of $k$ is much smaller in comparison to the quantity of items in use over all locations at the current group (i.e., $n$ ). We also assume that the percentages of repaired, remanufactured or replaced refrigerators being used at the current group are almost the same at all times. These two considerations lead us to model the failure of items using a deterministic failure rate $\lambda$. Before discussing the related operating policy in more detail, we present in Table 1 the notation used in the paper.

In this setting, the company incurs inventory holding costs for spare items, inventory holding costs for failed items at the warehouse, waiting costs due to the loss of goodwill of users whose failed items are yet to be replaced, and transportation costs. The problem is to find the optimum values of shipment cycle (i.e., $T$ ) and the level of spare items at the warehouse at the beginning of each cycle (i.e., $m$ ). We model this problem with the objective of jointly minimizing the long-run average total costs of the company under the truck capacity constraint (i.e., $T \lambda \leq P$ ) and the constraint that $T \lambda \leq k+m$. Note that, if the latter is violated, then the level of failed items in a cycle exceeds the level of spare items by more than $k$. This implies that all spare items are used to replace the failed items in a cycle, and yet, the level of failed items which cannot be immediately replaced exceeds $k$. If this constraint is satisfied, the level of operating items at the current group is at least $n-k$ during each shipment cycle.

In order to model and analyze this problem, we further consider the following cases:

(i) $m<T \lambda \leq k+m$ : This refers to the case where the level of spare items is less than the level of items that fail. Therefore, spare items can be used for immediate replacement of only a portion of failed items. At the end of the shipment cycle, there is an accumulated level of $T \lambda-m$ failed items which could not be immediately replaced.

(ii) $T \lambda \leq m$ : In this case, all failed items are immediately replaced with spare items and there is no waiting cost incurred.

Next, we provide expressions for long-run average total costs of the company in each of the above cases. For notational convenience we let $Q$ be equal to $T \lambda$, and treat $Q$ and $m$ as decision variables. It could be easily verified that if $m<T \lambda \leq k+m$, then the long-run average total costs are given by $T C_{1}(Q, m)$, where

$T C_{1}(Q, m)=\frac{m^{2} h_{1}}{2 Q}+\frac{Q h_{2}}{2}+\frac{(Q-m)^{2}}{2 Q} w+R \frac{\lambda}{Q}$.

An illustration of inventory levels of all items (in operating condition, failed and spare) and the demand level in this case is provided in Fig. 2. Similarly, if $T \lambda \leq m$, then the long-run average total costs are given by $T C_{2}(Q, m)$, where

$T C_{2}(Q, m)=\frac{(2 m-Q) h_{1}}{2}+\frac{Q h_{2}}{2}+R \frac{\lambda}{Q}$

An illustration of inventory levels of all items and the demand level corresponding to this case is provided in Fig. 3.

Observe that in both of the above cases, $Q$ is equal to the quantity of failed items during a shipment cycle. It is also the quantity of items that need to supplied from the central location to bring the level of items in working condition to a value of $n+m$ at the beginning of each cycle. If $m<T \lambda \leq n+m$, of these $T \lambda$ items, $m$ items are placed in the warehouse as spare, and $T \lambda-m$ are used to satisfy the demand of users who had failed items in the previous cycle. If $T \lambda \leq m$, all failed items in the previous cycle are immediately replaced with spare items. In this case, the replenishment quantity of $T \lambda$ items are placed in the warehouse as spare items.

Considering all the above cases, it turns out the company's long-run average total costs are given by

$T C(Q, m)= \begin{cases}T C_{1}(Q, m) & \text { if } m<Q \leq k+m \\ T C_{2}(Q, m) & \text { if } Q \leq m\end{cases}$

Notice from expression (2) that, for any value of $Q$ such that $Q \leq P$, the optimum value of $m$, under the constraint $Q \leq m$, is $Q$. Furthermore, the functional values of $T C_{1}(Q, m)$ and $T C_{2}(Q, m)$ at $Q=m$ are equal. Therefore, minimizing the long-run average total costs as defined in expression (3) is equivalent to minimizing $T C_{1}(Q, m)$ subject to $m \leq Q \leq k+m$ and all other constraints. Therefore, we reduce the company's optimization problem to the following:

$$
\begin{aligned}
\min \quad & T C(Q, m)=\frac{m^{2} h_{1}}{2 Q}+\frac{Q h_{2}}{2}+\frac{(Q-m)^{2}}{2 Q} w+R \frac{\lambda}{Q} \\
\text { s.t. } \quad & m \leq Q, \\
Q & \leq m+k, \\
Q & \leq P, \\
Q & >0, \\
& m \geq 0 .
\end{aligned}
$$

In the next section, we analyze the properties of the objective function and develop an algorithm to find the value of $Q$ and $m$ that jointly solve the above problem. We refer to these values as $Q^{*}$ and $m^{*}$. The optimum cycle length can in turn be found by using the relation $T^{*}=Q^{*} / \lambda$.

\section{Analysis of the problem}

We start with proving some useful properties of the objective function. These properties will be used later in this section to develop an algorithm to find $Q^{*}$ and $m^{*}$. 
Inventory level of operating items over al locations

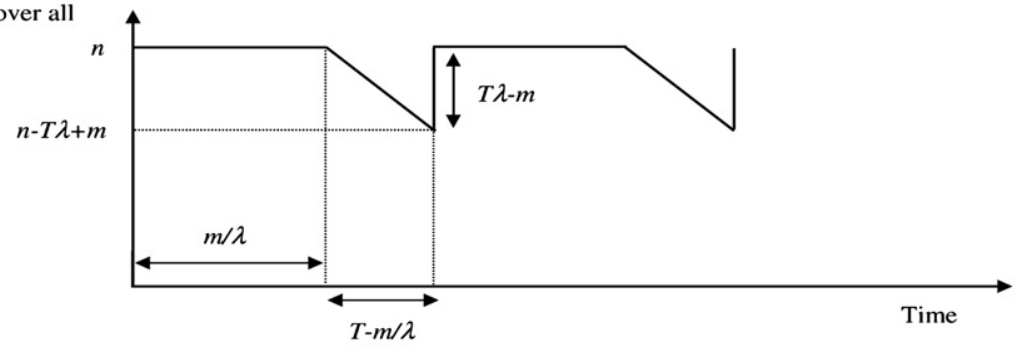

Inventory level of spare items at the warehouse

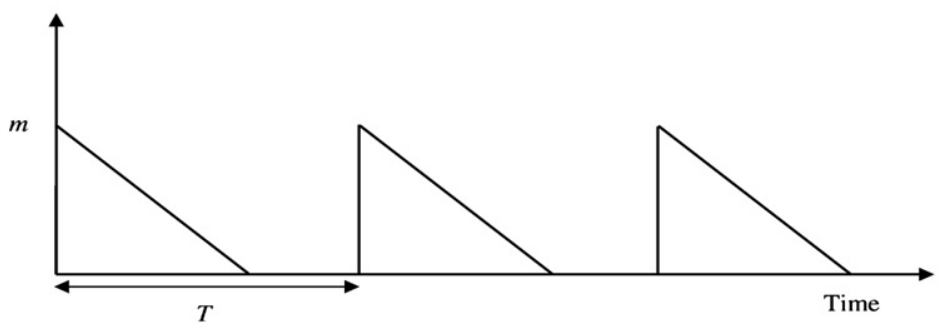

Inventory level of failed items at the warehouse

$T \lambda$

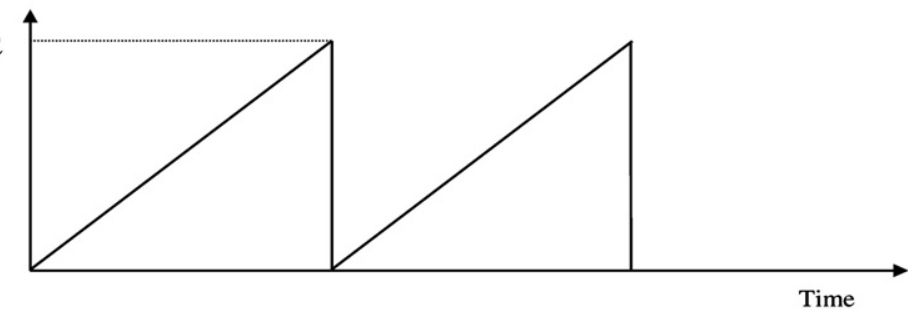

Level of demand waiting to be supplied from the next shipment

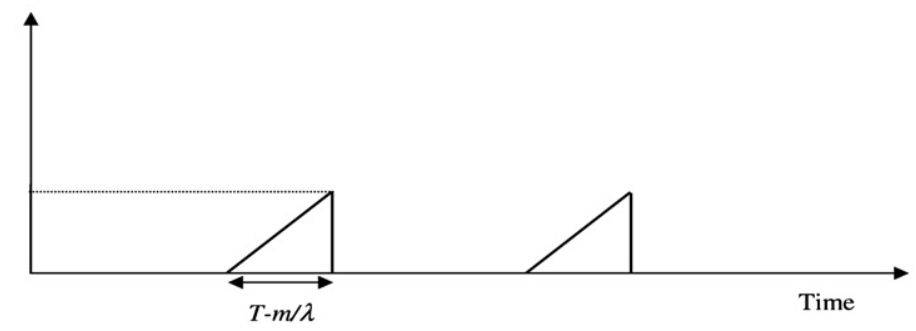

Fig. 2. An illustration of inventory levels in case of $m<T \lambda \leq k+m$.

Proposition 1. The long-run average total cost function $T C(Q, m)$ is convex over the region $Q>0, m \geq 0$, and, $\bar{Q}=$ $\sqrt{2 R \lambda\left(h_{1}+w\right) /\left(h_{1} h_{2}+h_{2} w+h_{1} w\right)}$ and $\bar{m}=w \bar{Q} /\left(h_{1}+w\right)$ jointly minimize this function in this region.

Proposition 1 implies that the values $\bar{Q}$ and $\bar{m}$ would jointly minimize the company's long- run average total costs if $m \leq Q$ was the only constraint besides $Q>0$ and $m \geq 0$. However, depending on the values of $k$ and $P$, these values may not be feasible, as implied by the second and third constraints of the company's optimization problem. The next corollary to Proposition 1 provides sufficient conditions for $\bar{Q}$ and $\bar{m}$ to be optimal.

Corollary 1. If $\bar{Q}$ satisfies $\bar{Q} \leq P$ and $\bar{Q} \leq k\left(\left(h_{1}+w\right) / h_{1}\right)$, then $Q^{*}=\bar{Q}$ and $m^{*}=\bar{m}$.

Proposition 1 provides a useful property of the objective function and Corollary 1 presents a sufficient condition for the joint optimality of $\bar{Q}$ and $\bar{m}$. In finding an optimal solution to the company's cost minimization problem, we will continue our analysis by dividing the feasible region into two parts, as defined by the following constraints: $Q \leq k\left(\left(h_{1}+w\right) / h_{1}\right)$ and $Q>k\left(\left(h_{1}+w\right) / h_{1}\right)$. We will find optimizers under these additional constraints, and form the final solution by comparing the costs that the optimizers in each region lead to.
Proposition 2. Let $Q_{1}^{*}$ and $m_{1}^{*}$ be the joint minimizers of the company's cost minimization problem under the additional constraint that $Q \leq k\left(\left(h_{1}+w\right) / h_{1}\right)$. The values of $Q_{1}^{*}$ and $m_{1}^{*}$ are given by

$Q_{1}^{*}=\min \left\{\bar{Q}, P, k\left(\frac{h_{1}+w}{h_{1}}\right)\right\}, \quad m_{1}^{*}=\frac{w Q_{1}^{*}}{h_{1}+w}$.

Proposition 3. Consider a case where $k\left(\left(h_{1}+w\right) / h_{1}\right)<P$. Let $Q_{2}^{*}$ and $m_{2}^{*}$ be the joint minimizers of the company's optimization problem under the additional constraint that $Q>k\left(\left(h_{1}+w\right) / h_{1}\right)$. The values of $Q_{2}^{*}$ and $m_{2}^{*}$ are given by

$Q_{2}^{*}=\min \left\{\max \left\{\sqrt{\frac{k^{2} h_{1}+k^{2} w+2 R \lambda}{h_{1}+h_{2}}}, k\left(\frac{h_{1}+w}{h_{1}}\right)+\varepsilon\right\}, P\right\}$,

$m_{2}^{*}=Q_{2}^{*}-k$,

where $\varepsilon$ is a positive and very small number.

Using Propositions 2 and 3, and comparing the cost at $Q_{1}^{*}$ and $m_{1}^{*}$ with the cost at $Q_{2}^{*}$ and $m_{2}^{*}$, one can obtain the optimal solution to the company's optimization problem. In case of $k\left(\left(h_{1}+w\right) / h_{1}\right) \geq P$, all values of $Q$ such that $Q>k\left(\left(h_{1}+w\right) / h_{1}\right)$ violate the truck capacity constraint. Therefore, the optimal solution in this case is given by the pair of $Q_{1}^{*}$ and $m_{1}^{*}$. We also note that, as $\varepsilon$ approaches to zero, the functional value of $T C(Q, m)$ at $Q=k\left(\left(h_{1}+w\right) / h_{1}\right)+\varepsilon$ and $m=Q-k$ approaches to the 
Inventory level of operating items over all locations

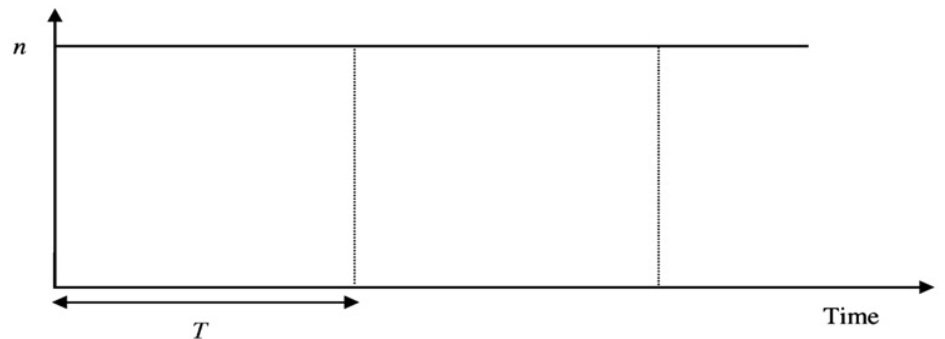

Inventory level of spare items at the warehouse

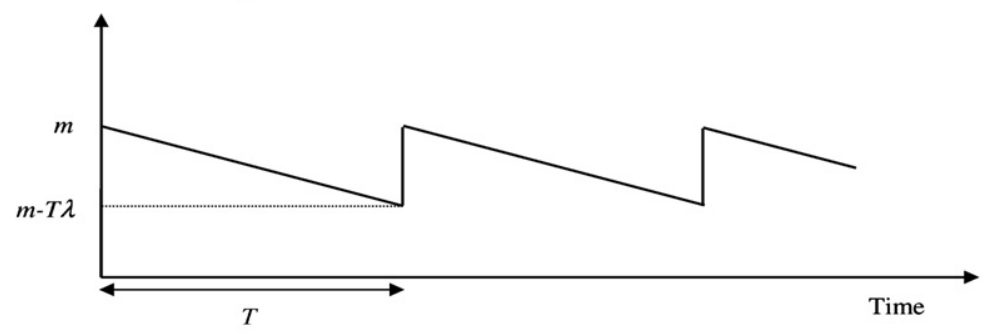

Inventory level of
failed items at the warehouse

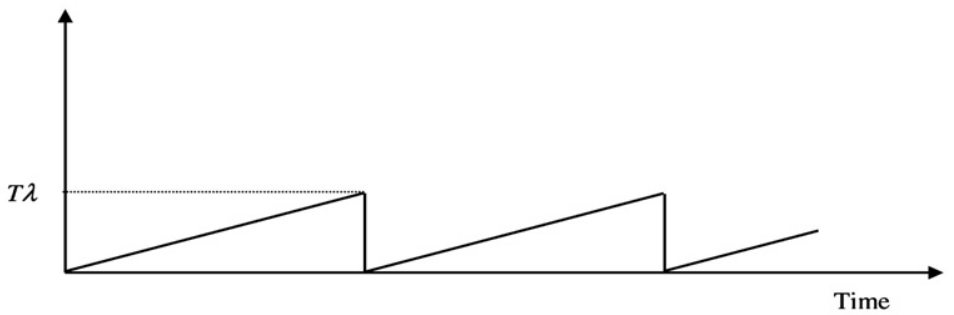

Level of demand waiting to be supplied from the next shipment

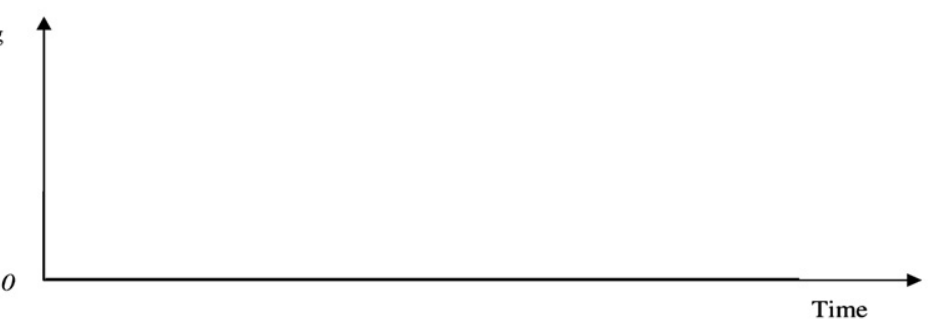

Fig. 3. An illustration of inventory levels in case of $T \lambda \leq m$.

functional value of $T C(Q, m)$ at $Q=k\left(\left(h_{1}+w\right) / h_{1}\right)$ and $m=$ $w Q /\left(h_{1}+w\right)$. Combining this fact with the following lemma, we will prove in Proposition 4 that as long as $k\left(\left(h_{1}+w\right) / h_{1}\right)$ is not equal to $\bar{Q}, \sqrt{\left(k^{2} h_{1}+k^{2} w+2 R \lambda\right) /\left(h_{1}+h_{2}\right)}$ or $P$, it cannot be optimal.

Lemma 1. We have $k\left(\left(h_{1}+w\right) / h_{1}\right)<\sqrt{\left(k^{2} h_{1}+k^{2} w+2 R \lambda\right) /\left(h_{1}+h_{2}\right)}$ if and only if $k\left(\left(h_{1}+w\right) / h_{1}\right)<\bar{Q}=\sqrt{\left(2 R \lambda\left(h_{1}+w\right)\right) /\left(h_{1} h_{2}+h_{2} w+h_{1} w\right)}$.

Propositions 2, 3 and Lemma 1 jointly lead to the following proposition which provides the optimal solution.

Proposition 4. Among the following values of $Q$ and $m$, the pair (i.e., $(Q, m))$ which is feasible and results in the least cost, optimizes the company's long-run average total costs: $(\bar{Q}, \bar{m}),\left(P, w P /\left(h_{1}+w\right)\right)$, $\left(\sqrt{\left(k^{2} h_{1}+k^{2} w+2 R \lambda\right) /\left(h_{1}+h_{2}\right)}, \sqrt{\left(k^{2} h_{1}+k^{2} w+2 R \lambda\right) /\left(h_{1}+h_{2}\right)}-k\right)$, $(P, P-k)$.

Proposition 4 implies that when truck capacity is small/ constraining, then the quantity shipped from the central location to the collection center at each cycle (i.e., $Q$ ) should be equal to the truck capacity. In this case, neither $Q$ nor $m$ depends on the failed-item inventory-holding-cost rate (i.e., $h_{2}$ ) or the failure rate (i.e., $\lambda$ ) or the per-truck cost $(R)$. The per-truck cost, failed-item inventory-holding-cost rate, and the failure rate affect the solution only if $P$ is not constraining. Independent of the truck capacity, if the measure for service level (i.e., $k$ ) is not small/ constraining, the ratio $w /\left(h_{1}+w\right)$ is critical in finding the number of spare items. More specifically, in this case, the number of spare items is a multiple of $Q$ by this ratio.

\section{Numerical results}

In this section, we will first show implementations of Corollary 1 and Proposition 4 to find the optimal pair of $Q^{*}$ and $m^{*}$ for some instances of the problem. Later, we will present the results of a numerical analysis to find out how the long-run average total cost at the optimal solution changes with respect to $k$ at varying levels of $w$. This will be followed by a numerical illustration of the impact of a wrong estimate for $\lambda$ on the actual cost incurred.

Example 1. In this example, four instances of the problem are presented to illustrate the possible forms of the solution as characterized by Proposition 4. These instances and the optimal solution in each case are summarized in Table 2.

In Table 2, each instance refers to a case where the solution is given by one of the possible solutions in Proposition 4, in the order they are presented in the proposition.

Recall that $k$ is an upper bound on the level of failed items in each cycle which cannot be immediately replaced and the users of 
Table 2

Instances of the problem illustrating possible forms of the solution.

\begin{tabular}{|c|c|c|c|}
\hline Instance number & Parameters & Optimal solution & Cost of optimal solution \\
\hline 1 & $h_{1}=20, h_{2}=15, w=25, P=5, R=30, \lambda=10, k=4$ & $Q^{*}=4.794, m^{*}=2.663$, and $T^{*}=0.479$ & $T C(4.794,2.663)=125.167$ \\
\hline 2 & $h_{1}=20, h_{2}=15, w=25, P=5, R=30, \lambda=20, k=4$ & $Q^{*}=5, m^{*}=2.778$, and $T^{*}=0.25$ & $T C(5,2.778)=185.277$ \\
\hline 3 & $h_{1}=27, h_{2}=15, w=9, P=40, R=30.8, \lambda=10, k=3$ & $Q^{*}=4.73, m^{*}=1.73$, and $T^{*}=0.473$ & $T C(4.73,1.73)=117.69$ \\
\hline 4 & $h_{1}=20, h_{2}=15, w=25, P=5, R=30, \lambda=20, k=2$ & $Q^{*}=5, m^{*}=3$, and $T^{*}=0.25$ & $T C(5,3)=185.5$ \\
\hline
\end{tabular}

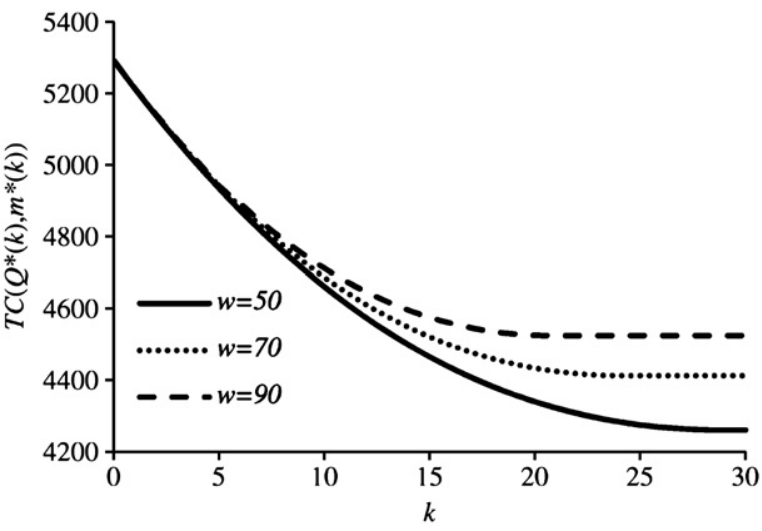

Fig. 4. Long-run average total cost at the optimal solution for varying values of $k$.

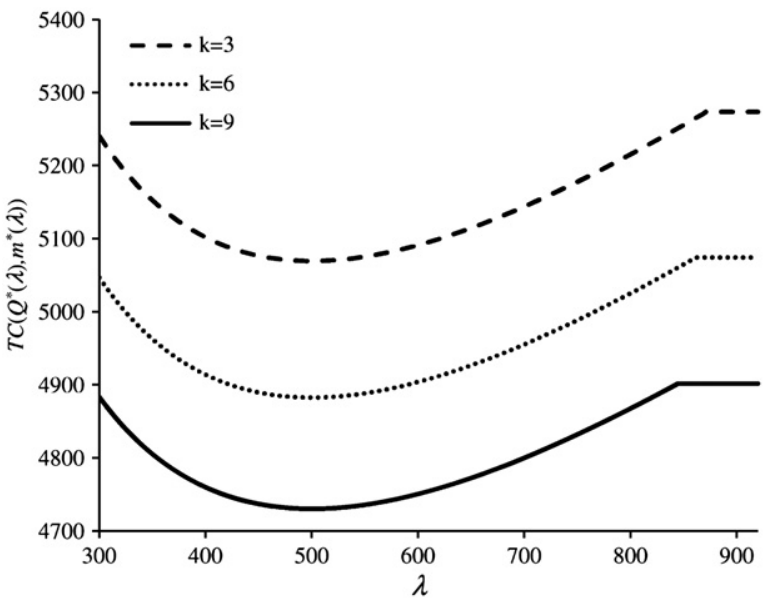

Fig. 5. Long-run average total cost at $\lambda=500$ given that the initial computation of $Q^{*}$ and $m^{*}$ was done using $\lambda$.

these items have to wait until the next shipment from the central depot. The objective of the next example is to investigate the effect of $k$ on the optimal solution. The optimal values of $Q$ and $m$ for a given $k$ value will be denoted by $Q^{*}(k)$ and $m^{*}(k)$, respectively.

Example 2. Consider a setting where $h_{1}=80, h_{2}=60, P=50$, $R=200, \lambda=500$. TC $\left(Q^{*}(k), m^{*}(k)\right)$ will be plotted with respect to $k$ at $w=50, w=70$ and $w=90$.

Fig. 4 shows a plot of the long-run average total cost at the optimal solution with respect to $k$ at three levels of $w$. Allowing $k$ to be a larger value, that is, loosening the service level constraint, helps to reduce costs initially by holding less amount of spare items in the local warehouse. However, for each $w$, there is a value of $k$ after which the long- run average total cost at the optimal solution does not change. In Example 2, increasing $k$ beyond 28.9, 24.2 and 20.8 when $w=50, w=70$ and $w=90$, respectively, does not change the optimal solution. Because, after these threshold values are reached, the savings in inventory holding costs due to holding less amount of spare items, does not justify the waiting costs of the users. Therefore, even if a larger value is allowed for $k$, the actual number of failed items which cannot be immediately replaced, i.e., $Q-m$, does not change in the optimal solution. Observe also that, the threshold values of $k$ after which the optimal solution does not change, are decreasing in $w$. Because, at larger values of $w$, waiting costs build up rapidly for those items which cannot be immediately replaced, therefore, the additional savings from inventory holding costs diminishes at smaller values of $k$.

The next example illustrates the impact of a wrong estimate for the failure rate $\lambda$ on the actual cost incurred.

Example 3. We revisit Example 2 by taking $w=70$ and consider the actual cost incurred by setting $\lambda$ to changing values within [300,920] despite its correct value of 500 .

Fig. 5 shows a plot of the actual long-run average total cost incurred (i.e., under $\lambda=500$ ) given that the initial computation of $Q$ and $m$ was done optimally but using a wrong value of $\lambda$. We refer to these values using the notation $Q^{*}(\lambda)$ and $m^{*}(\lambda)$. The longrun average total costs are plotted at three different values of $k$, those are 3, 6 and 9. If $\lambda$ was taken correctly, the actual long-run average total cost would be 5069.33, 4882.45 and 4729.86 for $k=3, k=6$ and $k=9$, respectively. As the value of $\lambda$ deviates from its correct value more, the actual cost incurred increases. The figure shows that taking a value of $\lambda$ slightly higher than 500 has a smaller impact on the actual cost in comparison to the case when $\lambda$ is lower than 500 by the same amount. Furthermore, the actual costs do not change after $\lambda=871, \lambda=862$ and $\lambda=845$ when $k=3$, $k=6$ and $k=9$, respectively. The reason is that initially $Q^{*}(\lambda)$ and $m^{*}(\lambda)$ at increasing values of $\lambda$ become larger, however, after these values of $\lambda$ (i.e., $\lambda=871, \lambda=862$ and $\lambda=845$ for $k=3, k=6$ and $k=9$ ) are reached, truck capacity happens to be binding on the quantity shipped from the central location to the warehouse. As a result, $Q^{*}(\lambda)$ and $m^{*}(\lambda)$ values do not change even if $\lambda$ increases any further, and therefore, the actual cost incurred remains the same.

\section{Conclusions}

In this paper, we place emphasis on explicitly modeling transportation costs and capacities in finding the economic shipment quantities of recovered and failed items between a central depot/ recovery location and a collection center/local warehouse, and the level of spare items to be held at the collection center. We consider a deterministic failure rate and a service level constraint. This constraint is defined in terms of the maximum level of failed items in each cycle which are not immediately replaced. Transportation costs and capacities are modeled using a multiple-setups cost structure.

The paper does not take into account the replenishment decisions for replacing the disposed items, or the inspection and separation procedures at the central depot. However, the multiple-setups cost structure allows the proposed model and its solution to be also used for the latter issue. More specifically, if inspection/separation at the 
central depot is done subject to a capacity restriction of $P$ items with a fixed cost of $R$ money units, the objective function corresponds to the sum of long-run average inventory holding costs at the collection center and the long-run average inspection/separation costs at the central depot. The analysis in the paper reduces the feasible solution to at most four different possibilities characterized by different parameters of the problem. The solution in each case depends on either the exact value of the truck cost $R$ or the truck capacity $P$.

The literature on remanufacturing and repairable items is very wide. The current study is related to the part of this literature that concerns management of inventories, and it contributes to this area by, first, modeling transportation costs and capacities, and secondly, by coordinating the shipment scheduling decisions of the failed and the serviceable items to save from transportation costs. In the literature, the general motivation for holding inventories of spare items at different locations is due to minimizing shortage costs or satisfying service levels during the repair times of the failed items. However, when there are transportation costs and capacities as in the current problem, it may not be advantageous to ship serviceable items (repaired or newly purchased) — even if they are ready_until an economic shipment quantity is reached. Therefore, spare items should be held at the collection center(s) to minimize shortage costs or to satisfy service levels during the waiting time for the next replenishment. In the current study, these issues are explicitly modeled. Furthermore, in a typical remanufacturing environment that consists of two stages (bases and depot), items are shipped in both directions. Under the existence of transportation costs and capacities, there is opportunity for savings if the material flow is coordinated to utilize the same vehicle in both directions. The current study is also unique in capturing this characteristic of a remanufacturing environment.

We would like to note that this study is a simplification of a real life problem, as described in more detail in Section 1. In the real problem, there are multiple collection centers. Therefore, routing the vehicles to consolidate items at several locations is also part of the solution. The current study focuses on part of this problem by considering a single collection center and a central depot. Focusing on this part of the larger problem, many tradeoffs, cost components that are relevant in the real case are captured in the proposed model, and yet, closed-form expressions for the possible solutions are found (see Proposition 4). These expressions clearly show the effects of the parameters on the solution. Moreover, they signify that transportation costs and capacities should in fact be taken into account.

The concept of coordinating the flows that appear in both directions in a remanufacturing environment in order to reduce transportation costs is an important one. This paper can be considered as a first-step analysis that can be extended to other settings including random failure rate of items or multiple collection centers, etc.

\section{Appendix A}

\section{A.1. Proof of Proposition 1}

We will first show that, for each pair of $Q$ and $m$ such that $m \geq 0$ and $Q>0$, all principal minors of the Hessian of $T C(Q, m)$ are nonnegative. This will prove the convexity. Then, we will show that the pair of $\bar{Q}$ and $\bar{m}$ constitutes a local minimum, and therefore, due to convexity, they minimize $T C(Q, m)$.

The Hessian is given by

$$
\left[\begin{array}{cc}
\frac{m^{2}\left(h_{1}+w\right)+2 R \lambda}{Q^{3}} & \frac{-m\left(h_{1}+w\right)}{Q^{2}} \\
\frac{-m\left(h_{1}+w\right)}{Q^{2}} & \frac{h_{1}+w}{Q}
\end{array}\right]
$$

The first principal minors of $T C(Q m)$ are given by $\partial^{2} T C$ $(Q, m) / \partial Q^{2}=\left(m^{2}\left(h_{1}+w\right)+2 R \lambda\right) / Q^{3}$ and $\partial^{2} T C(Q / \lambda, m) / \partial m^{2}=\left(h_{1}+\right.$ $w) / Q$. Because $Q>0, m \geq 0 h_{1}>0, w>0, R>0$, and $\lambda>0$, the first principal minors are nonnegative. The second principal minor is the determinant of the Hessian and equals $2 R \lambda\left(h_{1}+w\right) / Q^{4}$. Since this value is also nonnegative, $T C(Q, m)$ is convex in the region $Q>0$ and $m \geq 0$.

Now, let us consider the stationary points of $T C(Q m)$, those are the pairs of $Q$ and $m$ for which $\partial T C(Q, m) / \partial Q=0$ and $\partial T C(Q, m) /$ $\partial m=0$. We have

$\frac{\partial T C(Q, m)}{\partial Q}=-\frac{m^{2}\left(h_{1}+w\right)+2 R \lambda}{2 Q^{2}}+\frac{h_{2}+w}{2}$

and

$\partial T C(Q / \lambda, m) / \partial m=\frac{m h_{1}}{Q}+\frac{m w}{Q}-w$.

Setting these two expressions equal to zero and solving for $Q$ and $m$ lead to

$\bar{Q}=\sqrt{\frac{2 R \lambda\left(h_{1}+w\right)}{h_{1} h_{2}+h_{2} w+h_{1} w}}$

and

$\bar{m}=\frac{w \bar{Q}}{h_{1}+w}$.

Note that, since $\bar{Q}>0$ and $\bar{m}>0$, the first leading principal minor of the Hessian matrix evaluated at $(\bar{Q}, \bar{m})$, i.e., $\left(\bar{m}^{2}\left(h_{1}+w\right)+2 R \lambda\right) / \bar{Q}^{3}$, is positive. The second leading principal minor of the Hessian matrix evaluated at $(\bar{Q}, \bar{m})$, i.e., $2 R \lambda\left(h_{1}+w\right) / \bar{Q}$, is also positive. Therefore, $(\bar{Q}, \bar{m})$ is a local minimum. Furthermore, as $\bar{Q}>0$ and $\bar{m}>0,(\bar{Q}, \bar{m})$ is within the region where $T C(Q m)$ is convex. Hence, $\bar{Q}$ and $\bar{m}$ jointly minimize $T C(Q, m)$ over $Q>0$ and $m \geq 0$.

\section{A.2. Proof of Corollary 1}

From Proposition 1, we know that $\bar{Q}$ and $\bar{m}$ jointly minimize $T C(Q, m)$ over $Q>0$ and $m \geq 0$. If we can show that $\bar{Q}$ and $\bar{m}$, under the given conditions, are also feasible with respect to the other conditions, then this will prove their optimality for the overall optimization problem. Since $w /\left(h_{1}+w\right)<1$, it follows that $\bar{m}<\bar{Q}$, and hence, $\bar{Q}$ and $\bar{m}$ satisfy the first constraint (i.e., $m \leq Q$ ). The condition $\bar{Q} \leq P$ ensures that the truck capacity is not exceeded, and therefore, the third constraint is also trivially satisfied. If $\bar{Q} \leq k\left(\left(h_{1}+w\right) / h_{1}\right)$, then $\bar{Q} h_{1} /\left(h_{1}+w\right) \leq k$. This, in turn, implies that $\bar{Q}\left(1-w /\left(h_{1}+w\right)\right) \leq k$, and therefore, $w \bar{Q} /\left(h_{1}+w\right) \geq \bar{Q}-k$. Observe that, the left side of this inequality is given by $\bar{m}$, which leads to $\bar{m} \geq \bar{Q}-k$. Hence, under the given conditions, $\bar{Q}$ and $\bar{m}$ are feasible, and this implies their joint optimality.

\section{A.3. Proof of Proposition 2}

We know from Proposition 1 that under the constraints $m \geq 0$ and $Q>0$, the function $T C(Q, m)$ is convex. This implies that for fixed value of $Q, T C(Q, m)$ is convex with respect to $m$. Setting the first order partial derivative of $T C(Q, m)$ with respect to $m$ equal to zero and solving for $m$ leads to $w Q /\left(h_{1}+w\right)$ as its optimal value as a function of given $Q$. It can be easily shown that for any $Q$ such that $Q \leq k\left(\left(h_{1}+w\right) / h_{1}\right), m=w Q /\left(h_{1}+w\right)$ satisfies the constraint $Q \leq m+k$. Plugging this value in $T C(Q, m)$ leads to

$\frac{Q h_{1} w}{2\left(h_{1}+w\right)}+\frac{Q h_{2}}{2}+\frac{R \lambda}{Q}$.

Now, the company's optimization problem under $Q \leq k\left(\left(h_{1}+w\right) / h_{1}\right)$ is reduced to minimizing the above function with respect to the single variable $Q$ and, the constraints $Q \leq k\left(\left(h_{1}+w\right) / h_{1}\right)$ and $Q \leq P$. 
Observe that $\bar{Q}$ solves the unconstrained problem of minimizing expression (5). If $\bar{Q} \leq P$ and $\bar{Q} \leq k\left(\left(h_{1}+w\right) / h_{1}\right)$, then it is optimal. If $\bar{Q}>P$ or $\bar{Q}>k\left(\left(h_{1}+w\right) / h_{1}\right)$, then $T C\left(Q, w Q /\left(h_{1}+w\right)\right)$ is decreasing in the feasible region, therefore, the optimizer is given by the minimum of $P$ and $k\left(\left(h_{1}+w\right) / h_{1}\right)$.

\section{A.4. Proof of Proposition 3}

For any fixed value of $Q$ such that $Q>k\left(\left(h_{1}+w\right) / h_{1}\right)$, setting the first order partial derivative of $T C(Q, m)$ with respect to $m$ equal to zero and solving for $m$, we obtain $w Q /\left(h_{1}+w\right)$. The given value of $Q$ and $m=w Q /\left(h_{1}+w\right)$ satisfy the constraint $m \leq Q$. However, it turns out that for any $Q$ value such that $Q>k\left(\left(h_{1}+\right.\right.$ $\left.w) / h_{1}\right)$, the constraint $Q \leq m+k$ is violated at $m=w Q /\left(h_{1}+w\right)$. More specifically, we have $w Q /\left(h_{1}+w\right)<Q-k$. Due to the convexity of $T C(Q, m)$, this implies the function $T C(Q, m)$ is nondecreasing with respect to $m$ over $m \geq Q-k$, therefore, we choose $m=Q-k$. Plugging this value in $T C(Q, m)$ leads to

$\frac{Q\left(h_{1}+h_{2}\right)}{2}-k h_{1}+\frac{k^{2} h_{1}+k^{2} w+2 R \lambda}{2 Q}$.

Now, the company's optimization problem under $\left(Q>k\left(h_{1}+w\right) / h_{1}\right)$ is reduced to minimizing the above function with respect to the single variable $Q$ and, the constraints $\left(Q>k\left(h_{1}+w\right) / h_{1}\right)$ and $Q \leq P$. Observe that $\sqrt{\left(k^{2} h_{1}+k^{2} w+2 R \lambda\right) /\left(h_{1}+h_{2}\right)}$ solves the unconstrained problem of minimizing expression (6) with respect to $Q$. If $k\left(\left(h_{1}+w\right) / h_{1}\right)<P<\sqrt{\left(k^{2} h_{1}+k^{2} w+2 R \lambda\right) /\left(h_{1}+h_{2}\right)}$, then the function is nonincreasing over the feasible region, and therefore the minimizer is $P$. If $k\left(\left(h_{1}+w\right) / h_{1}\right)<\sqrt{\left(k^{2} h_{1}+k^{2} w+2 R \lambda\right) /\left(h_{1}+h_{2}\right)} \leq P$, then $\sqrt{\left(k^{2} h_{1}+k^{2} w+2 R \lambda\right) /\left(h_{1}+h_{2}\right)}$ is feasible and optimal. If $\sqrt{\left(k^{2} h_{1}+k^{2} w+2 R \lambda\right) /\left(h_{1}+h_{2}\right)} \leq k\left(\left(h_{1}+w\right) / h_{1}\right) \leq P$, then the function is nondecreasing over the feasible region, and therefore the minimizer is $k\left(\left(h_{1}+w\right) / h_{1}\right)+\varepsilon$, where $\varepsilon$ is a positive, very small number.

\section{A.5. Proof of Lemma 1}

If

$k\left(\frac{h_{1}+w}{h_{1}}\right)<\sqrt{\frac{k^{2} h_{1}+k^{2} w+2 R \lambda}{h_{1}+h_{2}}}$

then

$\frac{k^{2}\left(h_{1}^{2}+2 h_{1} w+w^{2}\right)}{h_{1}^{2}}<\frac{k^{2} h_{1}+k^{2} w+2 R \lambda}{h_{1}+h_{2}}$.

After some algebraic manipulations, this inequality can be rewritten as $k^{2}\left(h_{1}+w\right)\left(h_{1} h_{2}+h_{1} w+h_{2} w\right)<2 \lambda R h_{1}^{2}$, which implies $\left(k^{2}\left(h_{1}+w\right)^{2} / h_{1}^{2}\right)\left(h_{1} h_{2}+h_{1} w+h_{2} w\right)<2 \lambda R\left(h_{1}+w\right)$. This, in turn, leads to

$k\left(\frac{h_{1}+w}{h_{1}}\right)<\sqrt{\frac{2 R \lambda\left(h_{1}+w\right)}{h_{1} h_{2}+h_{2} w+h_{1} w}}$.

Similarly, it can be shown that if

$k\left(\frac{h_{1}+w}{h_{1}}\right)<\sqrt{\frac{2 R \lambda\left(h_{1}+w\right)}{h_{1} h_{2}+h_{2} w+h_{1} w}}$

then we have

$k\left(\frac{h_{1}+w}{h_{1}}\right)<\sqrt{\frac{k^{2} h_{1}+k^{2} w+2 R \lambda}{h_{1}+h_{2}}}$.

\section{A.6. Proof of Proposition 4}

Propositions 2 and 3 imply that, apart from the $(Q, m)$ pairs given in the current proposition, $\left(k\left(\left(h_{1}+w\right) / h_{1}\right), w k / h_{1}\right)$ and $\left(k\left(\left(h_{1}+w\right) / h_{1}\right)+\varepsilon, w k / h_{1}+\varepsilon\right)$ would be some candidates for optimal solution. Note that, due to the continuity of the objective function, the long-run average total costs at these two pairs get closer as $\varepsilon$ approaches to zero. The proof will follow by showing that if $Q_{1}^{*}=k\left(\left(h_{1}+w\right) / h_{1}\right)$, then a value of $Q_{2}^{*}$ different than $k\left(\left(h_{1}+w\right) / h_{1}\right)+\varepsilon$ gives lower costs. Similarly, if $Q_{2}^{*}=k\left(\left(h_{1}+w\right) /\right.$ $\left.h_{1}\right)+\varepsilon$, then a value of $Q_{1}^{*}$ different than $k\left(\left(h_{1}+w\right) / h_{1}\right)$ gives lower costs. Therefore, neither of them can be optimal.

Now, let us assume that $Q_{1}^{*}=k\left(\left(h_{1}+w\right) / h_{1}\right)$. Proposition 2 implies that $k\left(\left(h_{1}+w\right) / h_{1}\right)<\bar{Q}$. Combining this with Lemma 1 leads to $k\left(\left(h_{1}+w\right) / h_{1}\right)<\sqrt{\left(k^{2} h_{1}+k^{2} w+2 R \lambda\right) /\left(h_{1}+h_{2}\right)}$, which in turn, implies that $k\left(\left(h_{1}+w\right) / h_{1}\right)+\varepsilon \leq \sqrt{\left(k^{2} h_{1}+k^{2} w+2 R \lambda\right) /\left(h_{1}+h_{2}\right)}$ as $\varepsilon$ approaches to zero. Proposition 3 further leads to the fact that, in this case, $Q_{2}^{*}$ is either given by $P$ or $\sqrt{\left(k^{2} h_{1}+k^{2} w+2 R \lambda\right) /\left(h_{1}+h_{2}\right)}$, and $\left(Q_{2}^{*}, Q_{2}^{*}-k\right)$ results in lower costs than $\left(k\left(\left(h_{1}+w\right) / h_{1}\right)+\varepsilon, w k /\right.$ $\left.h_{1}+\varepsilon\right)$. We know that the costs at $\left(k\left(\left(h_{1}+w\right) / h_{1}\right)+\varepsilon, w k / h_{1}+\varepsilon\right)$ are approximately equal to the costs at $\left(k\left(\left(h_{1}+w\right) / h_{1}\right), w k / h_{1}\right)$ for very small values of $\varepsilon$. Therefore, $Q_{1}^{*}$ cannot be optimal.

Now, let us assume that $Q_{2}^{*}=k\left(\left(h_{1}+w\right) / h_{1}\right)+\varepsilon$. Proposition 3 implies that $k\left(\left(h_{1}+w\right) / h_{1}\right)+\varepsilon>\sqrt{\left(k^{2} h_{1}+k^{2} w+2 R \lambda\right) /\left(h_{1}+h_{2}\right)}$. Combining this with Lemma 1 leads to $k\left(\left(h_{1}+w\right) / h_{1}\right)+\varepsilon>\bar{Q}$, which in turn, implies that $k\left(\left(h_{1}+w\right) / h_{1}\right) \geq \bar{Q}$ as $\varepsilon$ approaches to zero. Proposition 2 further leads to the fact that, in this case, $Q_{1}^{*}$ is either given by $P$ or $\bar{Q}$, and $\left(Q_{1}^{*}, w Q_{1}^{*} /\left(h_{1}+w\right)\right)$ results in lower costs than $\left(k\left(\left(h_{1}+w\right) / h_{1}\right), w k / h_{1}\right)$. We know that the costs at $\left(k\left(\left(h_{1}+w\right) / h_{1}\right)+\varepsilon, w k / h_{1}+\varepsilon\right)$ are approximately equal to the costs at $\left(k\left(\left(h_{1}+w\right) / h_{1}\right), w k / h_{1}\right)$ for very small values of $\varepsilon$. Therefore, $Q_{2}^{*}$ cannot be optimal.

\section{References}

Aucamp, D.C., 1982. Nonlinear freight costs in the EOQ problem. European Journal of Operational Research 9, 61-62.

Burwell, T.H., Dave, D.S., Fitzpatrick, K.E., Roy, M.R., 1997. Economic lot size model for price-dependent demand under quantity and freight discounts. International Journal of Production Economics 48, 141-155.

Çetinkaya, S., Lee, C.-Y., 2002. Optimal outbound dispatch policies modeling inventory and cargo capacity. Naval Research Logistics 49, 531-556.

Guide, V.D.R., Srivastava, R., 1997. Repairable inventory theory: models and applications. European Journal of Operational Research 102, 1-20.

Kennedy, W.J., Patterson, J.W., Fredendall, L.D., 2002. An overview of recent literature on spare parts inventories. International Journal of Production Economics 76, 201-215.

Kim, J.-S., Shin, K.-C., Park, S.-K., 2000. An optimal algorithm for repairable-item inventory system with depot spares. Journal of the Operational Research Society 51, 350-357.

Koh, S.-G., Hwang, H., Sohn, K.-I., Ko, C.-S., 2002. An optimal ordering and recovery policy for reusable items. Computers and Industrial Engineering 43, 59-73.

Lee, C.-Y., 1989. A solution to the multiple set-up problem with dynamic demand. IIE Transactions 21, 266-270.

Mendoza, A., Ventura, J.A., 2008. Incorporating quantity discounts to the EOQ model with transportation costs. International Journal of Production Economics $113,754-765$.

Moinzadeh, K., Lee, H.L., 1986. Batch size and stocking levels in multiechelon repairable systems. Management Science 32, 1567-1581.

Nahmias, S., Rivera, H., 1979. A deterministic model for a repairable item inventory system with a finite repair rate. International Journal of Production Research 17, 215-221.

Richter, K., 1996a. The EOQ repair and waste disposal model with variable setup numbers. European Journal of Operational Research 96, 313-324.

Richter, K., 1996b. The extended EOQ repair and waste disposal model. International Journal of Production Economics 45, 443-447.

Rieksts, B.Q., Ventura, J.A., 2010. Two-stage inventory models with a bi-model transportation cost. Computers and Operations Research 37, 20-31.

Schrady, D.A., 1967. A deterministic inventory model for repairable items. Naval Research Logistics Quarterly 14, 391-398.

Sherbrooke, C.C., 1968. Metric: a multi-echelon technique for recoverable item control. Operations Research 16, 122-141. 
Teunter, R.H., 2001. Economic ordering quantities for recoverable item inventory systems. Naval Research Logistics 48, 484-495.

Tersine, R.J., Barman, S., 1994. Optimal lot sizes for unit and shipping discount situations. IIE Transactions 26, 97-101.

Toptal, A., 2009. Replenishment decisions under an all-units discount schedule and stepwise freight costs. European Journal of Operational Research 198, 504-510.
Toptal, A., Cetinkaya, S., Lee, C.-Y., 2003. The buyer-vendor coordination problem: modeling inbound and outbound cargo capacity costs. IIE Transactions 35, 987-1002.

Toptal, A., Çetinkaya, S., 2006. Contractual agreements for coordination and vendor-managed delivery under explicit transportation considerations. Naval Research Logistics 53, 1-21. 